\title{
Acquisition method of asphalt pavement texture information based on the CPR Technology
}

\author{
Chen Jiaying ${ }^{1}$, Zheng Binshuang ${ }^{1}$, Chen $\mathrm{Xi}^{1}$, Zhao Runmin ${ }^{1}$, Huang Xiaoming ${ }^{1, *}$ \\ ${ }^{1}$ School of Transportation, Southeast University, Nanjing 211189, China
}

\begin{abstract}
In order to obtain the asphalt pavement texture information in real time and accurately monitor the anti-skid performance of the road pavement, an automatic close range photogrammetry system (ACPR system) was proposed and built based on the circle arranged three cameras close range photogrammetry (CPR) technology to obtain the asphalt pavement surface texture. Automatic image acquisition and 3D reconstruction were achieved by the ACPR system. Sand patch method and laser scanning method (ZGScan) were used to collect the on-site comparison test of the asphalt pavement texture. Mean texture depth (MTD) and root mean square roughness (RSMR) were chosen as the statistical indicators of road surface texture. The results show that the texture data obtained by ACPR system has relatively high accuracy and efficiency, and the recognition accuracy is close to $0.02 \mathrm{~mm}$. The ACPR system improves the efficiency and accuracy of traditional close range photogrammetry and provides real-time and effective road surface anti-skid information for subsequent safety braking of autonomous vehicle.
\end{abstract}

Key words: Anti-skid performance; automated close range photogrammetry system; asphalt pavement texture information; mean texture depth (MTD); root mean square roughness (RMSR)

\section{Introduction}

In recent years, numerous highway traffic accidents happened due to the lack of asphalt pavement anti-skid performance. It is known that good pavement anti-skid performance can provide high-speed vehicles with sufficient friction to ensure the safety and comfort of the vehicle during driving. Anti-skid performance of asphalt pavement relates to road surface texture directly, especially on rainy days, reasonable asphalt pavement texture can better discharge water, penetrate the water film, thereby reducing the traffic accidents due to water drift slipping. Therefore, it is of great significance to periodically monitor the surface texture of asphalt pavement during the whole life cycle and obtain the anti-skid performance of asphalt pavement in time [1,2].

The earliest method of obtaining the surface texture of asphalt pavement is the contact measurement [3], which indirectly evaluates the surface texture of pavement by reading relevant index of instrument or calculating the texture depth and coefficient of friction. During the test, the traffic in the measurement site is affected, and the detailed information about the

Author: Chen Jiaying, cjiaying14@seu.edu.cn

* Corresponding author: Huang Xiaoming, huangxm@seu.edu.cn macroscopic texture and microscopic texture of the asphalt pavement is not available. Based on the above research, the non-contact measurement has been explored and researched by scholar world widely. Non-contact measurement mainly includes digital gray image [4], industrial CT scanning $[5,6]$, laser measurement, close range photogrammetry. These methods are involved in digital reconstruction of the road surface texture three-dimensional model. Compared with other non-contact measurement methods, the close range photogrammetry (CRP) method has the advantages of reducing test time and improving measurement efficiency [7,8]. In order to have sufficient overlap between the captured images, the traditional close range photogrammetry needs to capture more than 6 pictures around the object to be measured [9]. The number of image acquisition greatly affects the measurement efficiency, especially the processing time of post-image and operation time of three-dimensional modeling [10]. In order to improve the efficiency of obtaining the surface texture of asphalt pavement by the method of CRP, some scholars use the close range photogrammetry method based on dual camera to collect the surface texture of asphalt mixture specimen [7]. Although the 
mentioned method can improve the measurement efficiency, loss of texture information is unavoidable. Therefore, it is urgent to improve the traditional close range photogrammetry technology, and improve the precision and efficiency of texture reconstruction while guaranteeing the integrality of texture information.

Based on this, this paper proposes a close range photogrammetry method based on circle arranged three cameras. The ACRP system realizes the texture image collection and three-dimensional model reconstruction of asphalt pavement surface. MATLAB and Python are used to create three-dimensional reconstruction software module, which controls the CRP platform illumination and image acquisition process, and performs calculation of digital information and related texture parameters of surface texture of asphalt pavement. The ACPR system is used to test the texture information of asphalt pavement in site, and the surface texture parameters obtained by laser scanning method (ZGScan) and sand patch method are compared with those of ACRP system.

\section{ACRP system}

The close range photogrammetry can be divided into three major processes, including close range photography, image processing and 3D reconstruction [10]. After the image acquisition, a series of processing and operation of the image is needed to complete the three-dimensional reconstruction.

\subsection{Basics of close range photogrammetry}

Close range photogrammetry is based on binocular stereo vision technology which obtains images with time series of the same target at different points of time or different positions. By analyzing the geometric constraints of the image sequence, the three-dimensional coordinate information of target surface can be calculated. The principle is shown in Figure 1.

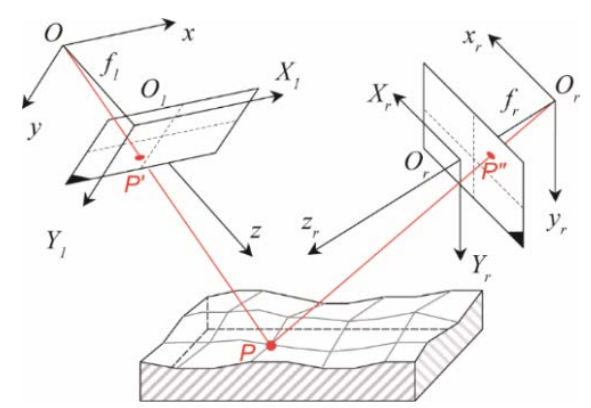

Fig. 1. Fundamentals of close range photogrammetry

Assume the camera is located at the origin of the world coordinate system $O-x y z$ at the initial moment, the captured image is in the coordinate system $O_{1}-X_{1} Y_{1}$, and the effective focal length of the camera is $f_{1}$. The camera coordinate system is $O_{r}-x_{r} y_{r} z_{r}$ at moment 2, the image coordinate system is $O_{r}-X_{r} Y_{r}$, the effective focal length is $f_{r}$. According to camera perspective transformation model, the formula can be obtained, as shown below:

$$
\begin{gathered}
s_{1}\left[\begin{array}{c}
X_{1} \\
Y_{1} \\
1
\end{array}\right]=\left[\begin{array}{lll}
f_{1} & 0 & 0 \\
0 & f_{1} & 0 \\
0 & 0 & 1
\end{array}\right]\left[\begin{array}{l}
x \\
y \\
z
\end{array}\right] \\
s_{r}\left[\begin{array}{c}
X_{r} \\
Y_{r} \\
1
\end{array}\right]=\left[\begin{array}{ccc}
f_{r} & 0 & 0 \\
0 & f_{r} & 0 \\
0 & 0 & 1
\end{array}\right]\left[\begin{array}{l}
x_{r} \\
y_{r} \\
z_{r}
\end{array}\right]
\end{gathered}
$$

Where, the relationship between the coordinate system $O-x y z$ and the coordinate system $O_{r}-x_{r} y_{r} z_{r}$ is transformed by the spatial matrix $M_{1 r}, \rho_{r}$ is the coordinates of the target space point $\mathrm{P}$ (see Figure 1) on the image of the subsequent time series. Therefore, the mathematical model of three-dimensional coordinates of target space point is established:

$$
\begin{aligned}
x & =z X_{1} / f_{1} \\
y & =z Y_{1} / f_{1} \\
z & =\frac{f_{1}\left(f_{r} t_{x}-X_{r} t_{z}\right)}{X_{r}\left(r_{7} X_{1}+r_{8} Y_{1}+f_{1} r_{9}\right)-f_{r}\left(r_{1} X_{1}+r_{2} Y_{1}+f_{1} r_{3}\right)} \\
& =\frac{f_{1}\left(f_{r} t_{y}-Y_{r} t_{z}\right)}{Y_{r}\left(r_{7} X_{1}+r_{8} Y_{1}+f_{1} r_{9}\right)-f_{r}\left(r_{4} X_{1}+r_{5} Y_{1}+f_{1} r_{6}\right)}
\end{aligned}
$$

The focal length $f_{1}, f_{r}$, coordinates $P^{\prime}$ and $P^{\prime \prime}$ of the target space point $\mathrm{P}$ on images have been known, inputting $R$ which is the rotation matrix of every two camera positions and the translation vector $T$, then the three-dimensional space coordinates of the target point $P$ can be calculated. 


\subsection{Establishment of ACRP system}

In order to improve the efficiency of traditional close range photogrammetry, this paper builds an ACRP system, which includes close range photogrammetry platform and three-dimensional reconstruction software module.

\subsubsection{Close range photographic platform}

The close range photographic platform comprises three Basler industrial cameras and a circular 60LED shadowless lamp illumination module, which are connected with the master computer via the USB3.0 data cable. Then use the camera API program on the master computer to send the command, which controls shadowless lamp module to provide illumination, and trigger three cameras to complete the acquisition of images at the same time, which can collect three images each time [11]. Camera parameters are shown in Table 1.

Table 1 Camera parameter settings

\begin{tabular}{cc|cc}
\hline Specifications & Properties & Specifications & Properties \\
\hline Camera model & Basler acA1300 & Frame Rate & $60 \mathrm{FPS}$ \\
& PYTHON 5000 & Lense model & Basler Lens \\
Sensor type & CMOS & & C125 \\
& $500 \times 10^{4}$ & Focal length & $8.0 \mathrm{~mm}$ \\
Effective pixels & Global Shutter & Aperture & F1.8-F22.0 \\
Shutter & $1 / 4,000 \mathrm{~s}$ & & \\
Shutter speed & & & \\
\hline
\end{tabular}

Note: CMOS: Complementary Metal Oxide Semiconductor. CMOS technology is used for several analog circuits such as image sensors.

The size of the close range photographic platform is $1 \times 1 \times 0.5 \mathrm{~m}(\mathrm{~L} \times \mathrm{W} \times \mathrm{H})$, the three cameras on the platform are arranged in a circular trajectory of $0.5 \mathrm{~m}$ radius with $120^{\circ}$ interval. The shadowless lamp illumination system is installed at the center of the circle, the shadowless lamp power is $60 \mathrm{~W}$, the brightness adjustment range is $0 \sim 4000 \mathrm{LX}$, The detailed structure and equipment of the ACRP System platform are shown in Figure 2.

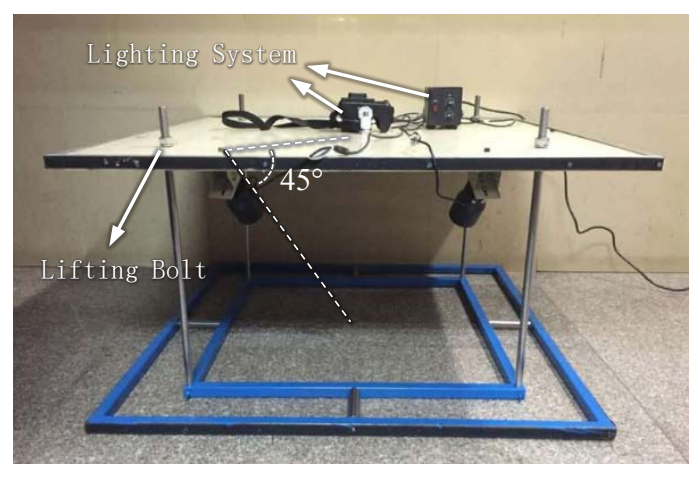

Fig. 2. Structure of ACRP System platform

\subsubsection{D reconstruction software module}

Through MATLAB and Python mixed programming to complete the three-dimensional reconstruction software module, the function of the module includes control of the CRP platform lighting and image acquisition. Meanwhile, complete the subsequent image processing and three-dimensional reconstruction to obtain the asphalt pavement surface texture digitization information and related texture parameters. Three-dimensional reconstruction module interface is shown in Figure 3.

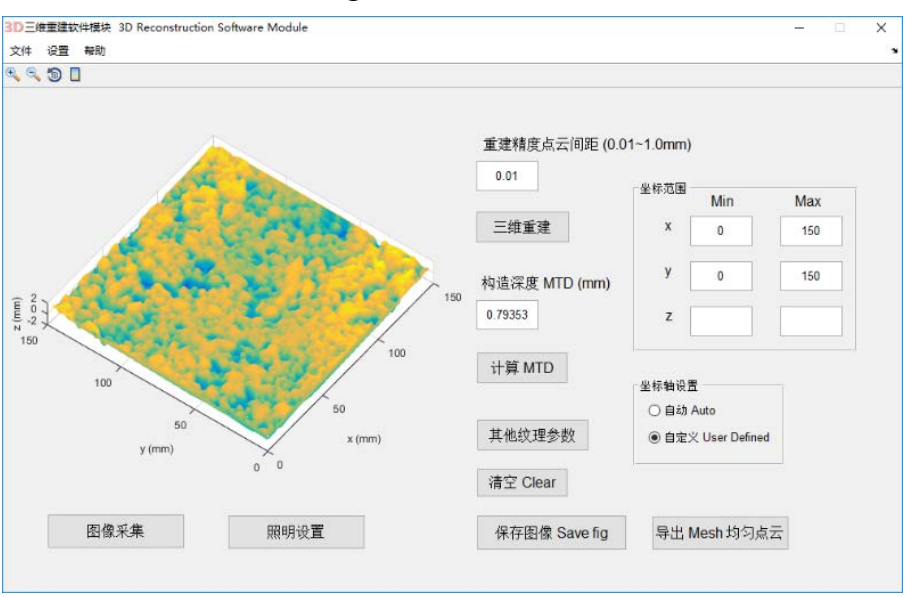

Fig. 3. 3D reconstruction software module

The following steps are required for the process of the software module design:

(1) Camera calibration and external parameter input

On the CRP platform, the camera external parameters such as the matrix $\mathrm{R}, \mathrm{T}$ of the camera coordinate system are obtained by camera calibration, fixed camera angle and azimuth. The camera external parameters are used as the known input parameters to calculate the spatial coordinate points [12]. 
(2) Image adjustment

Image adjustment includes image distortion elimination [13] and adjustment of image brightness, contrast. Image distortion elimination model is:

$$
\begin{aligned}
& \Delta x=\left(x-x_{0}\right)\left[k_{1} r^{2}+k_{2} r^{4}\right]+p_{1}\left[r^{2}+2\left(x-x_{0}\right)^{2}\right] \\
& +2 p_{2}\left(x-x_{0}\right)\left(y-y_{0}\right) \\
& \Delta y=\left(y-y_{0}\right)\left[k_{1} r^{2}+k_{2} r^{4}\right]+p_{2}\left[r^{2}+2\left(y-y_{0}\right)^{2}\right] \\
& +2 p_{1}\left(x-x_{0}\right)\left(y-y_{0}\right)
\end{aligned}
$$

Among them, $\Delta x, \Delta y$ are the image point displacement caused by the distortion of images, $\left(x_{0}, y_{0}\right)$ is the image main point coordinate (intersection of the perpendicular of the center of photography and the image plane and the image plane). $r^{2}=\left(x-x_{0}\right)^{2}+\left(y-y_{0}\right)^{2}, k_{1}, k_{2}, p_{1}, p_{2}$ are the radial distortion coefficient, and they are known parameters of the lens.

\section{(3) Point cloud reconstruction and registration}

Based on the matching of feature points between images, the coordinates of space point cloud data [14] are calculated by the SFM algorithm. If the coordinate of projection point $g_{i n}$ of space point $G_{\mathrm{i}}$ on the two-dimensional coordinate system where the camera seat is located in, is $\left(x_{i n}, y_{i n}\right)$, then the projection of all spatial points set $\mathrm{G}$ in $n$th frame image are:

$$
\left(\begin{array}{ccc}
x_{i 1} & \cdots & x_{i n} \\
y_{i 1} & \cdots & y_{i n}
\end{array}\right)=R\left[\begin{array}{ccc}
X_{1} & \cdots & X_{i} \\
Y_{1} & \cdots & Y_{i} \\
Z_{1} & \cdots & Z_{i}
\end{array}\right]+T
$$

Among them, $\left(X_{i}, Y_{i}, Z_{i}\right)$ is the three-dimensional coordinate of the space point $\mathrm{Gi}, R$ is the rotation matrix of every two camera positions and $T$ is the translation vector. They can be obtained by matching the feature points between the images (see section 1.1). Assume the minimum spacing between point cloud data is $0.01 \mathrm{~mm}$, the process of point cloud reconstruction to obtain a spatial point three-dimensional coordinate is shown in Figure 4. At the same time of point cloud reconstruction, the point cloud registration algorithm based on Iterative Closest Point (ICP) is used to register the point clouds, and the points cloud is transformed into the same coordinate system. Then search for an optimal geometric transformation that allows multiple point cloud data to be convergent in the same coordinate system as good as possible.

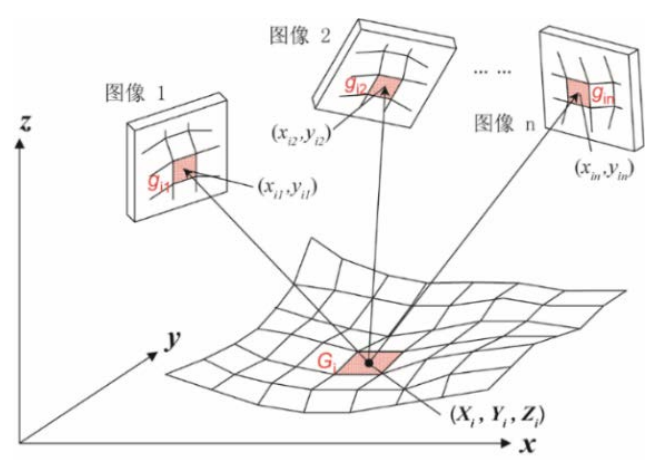

Fig. 4. Point cloud reconstruction to obtain the $3 D$ coordinate of space point

\section{(4) Generate 3D Texture model}

After completing the calculation of the cloud points (figure (a)), the triangular meshing is done (figure (b)) and the 3D texture model (figure (c) (d)) is generated. For the three-dimensional texture model, the model scale needs to be further adjusted. The local axis properties need to be defined. Hole filling correction and plane leveling are required as well.

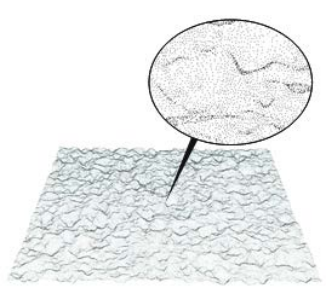

(a) Cloud point

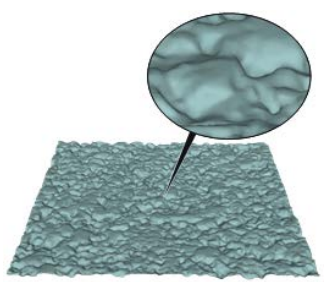

(c) 3D pavement model

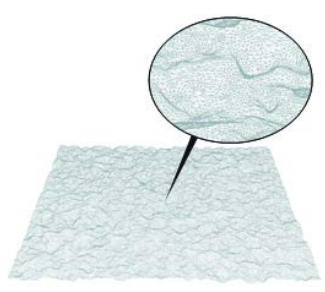

(b) Grid

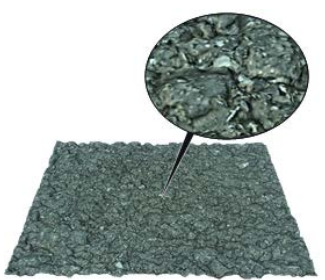

(d) 3D texture model
Fig. 5. Generating process of 3D pavement texture model

\section{Field testing}

Select Nanjing Jiangning District Liangjiang East Road for field testing, Liangjiang East Road is a two-way two-lane road (South-North direction /north-south direction), asphalt mixture grade is AC-13. 


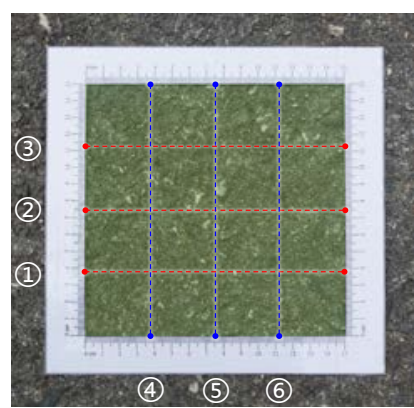

(a) Measuring range

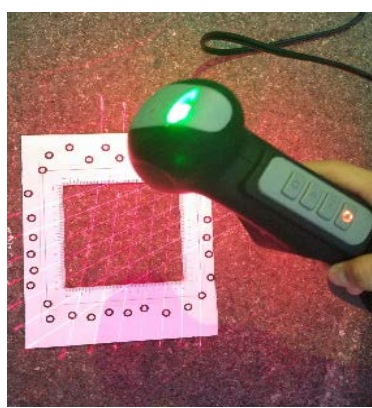

(b) Laser measurement

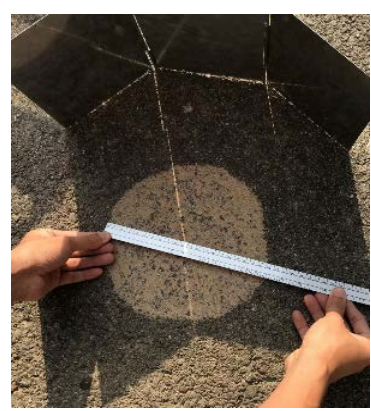

(c) Sand patch method

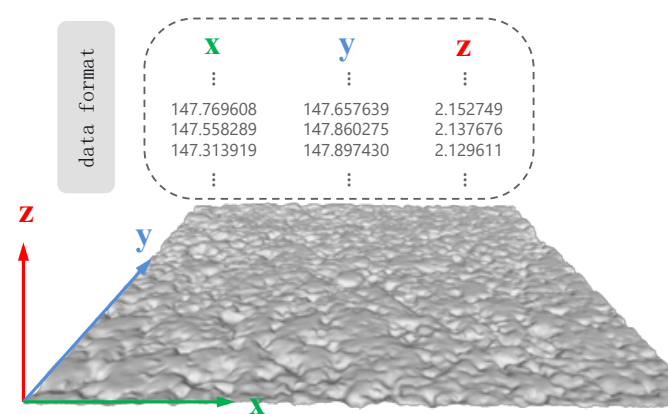

(d) Data format

Fig. 6. Measuring range and data format reconstruction

\subsection{Data acquisition}

The surface texture parameters were collected using ACRP System, laser measurement (Fig. 6(b)) and sand-patch method (Fig.6(c)). For the close range photogrammetry, the paper uses the proposed ACPR system to complete the road image acquisition and three-dimensional reconstruction. The test uses the ZGScan 717 PLUS industrial handheld laser scanner to obtain a three-dimensional model of the object to be tested directly, with an accuracy of $0.02 \mathrm{~mm}$ (Figure 6 (b)).

The three-dimensional model of the obtained pavement is processed and analyzed, including the fill hole correction, leveling, defining the local axis properties. Then exporting the texture three-dimensional elevation data (Figure 6 (d)) including $x y z$ 3D coordinates from the three-dimensional model of the pavement texture model. Texture elevation data with the same format can be obtained by using a post-processing procedure similar to that of the close range photogrammetry.

\subsection{Anti-skid evaluation index}

In this paper, two texture parameters including the mean texture depth (MTD) and root mean square roughness (RSMR) are selected to evaluate the asphalt pavement surface anti-skid performance. The calculation coverage of MTD is shown in Fig.6 (a), which is a $150 \mathrm{~mm} \times 150 \mathrm{~mm}$ square area. The calculation profiles of RSMR are indicated by the dashed lines in Fig 6 (a), which are $25 \%, 50 \%, 75 \%$ of the edge length, 6 profiles in total.
The mean texture depth is calculated as:

$$
\begin{gathered}
V=\iint_{D}\left[F_{0}-F(x, y)\right] d x d y \\
M T D=\frac{V}{A}
\end{gathered}
$$

Where, $F_{0}$ is the space plane covering on the road surface, $F(x, y)$ is the area formed by the elevation point of the pavement, $D$ is the integral area, $V\left(\mathrm{~mm}^{3}\right)$ is the volume enclosed between the road pavement and the plane $F_{0}$, and $A\left(\mathrm{~mm}^{2}\right)$ is the area of the integral area $D$.

The formula for calculating the root mean square roughness (RSMR) is:

$$
R M S R=\sqrt{\frac{1}{N} \sum_{i=1}^{N}\left[z\left(x_{i}\right)\right]^{2}}
$$

Where, $z\left(x_{i}\right)$ is the profile elevation point shown in Figure 6(b). Considering the accuracy of the road surface texture measurement results, the pavement texture is first tested by close range photogrammetry and laser measurement, and then the MTD value of the road pavement is measured by the sand patch method.

\section{Method validation}

\subsection{Accuracy of close range photogrammetry system}

The pavement texture parameters obtained by the laser measurement (ZGScan) and the sand patch method are compared with those of the CRP system, as shown in Tables 2 and 3. It can be seen from Table 2 that based on the results from the ZGScan handheld industrial laser scanner, the RSMR index obtained by the ACRP system 
basically reaches the ZGScan accuracy. The measurement results from the ACRP system are larger on the whole, and the mean relative error (MRE) is $-0.29 \%$, $-0.31 \%,-0.14 \%$, respectively.

Table 2. Calculation results of statistical index RSMR of asphalt pavement texture parameters

\begin{tabular}{|c|c|c|c|c|c|c|c|c|}
\hline \multirow{3}{*}{$\mathrm{P}$} & \multirow{3}{*}{ 3D Texture } & \multirow{3}{*}{ Profile } & \multicolumn{2}{|c|}{$\operatorname{RSMR}(\mathrm{mm})$} & \multirow{2}{*}{\multicolumn{4}{|c|}{ Statistical Parameter }} \\
\hline & & & \multirow{2}{*}{$\begin{array}{c}\text { Benchmark } \\
\text { ZGScan }\end{array}$} & \multirow{2}{*}{$\frac{\text { Validation }}{\text { ACRP System }}$} & & & & \\
\hline & & & & & $\mathrm{AE}(\mathrm{mm})$ & $\operatorname{MAE}(\mathrm{mm})$ & $\mathrm{RE}$ & MRE \\
\hline \multirow[t]{6}{*}{1} & & (1) & 0.0982 & 0.0986 & $-4.0 \mathrm{E}-04$ & $-2.83 \mathrm{E}-04$ & $-0.41 \%$ & $-0.29 \%$ \\
\hline & & (2) & 0.1107 & 0.1100 & $7.0 \mathrm{E}-04$ & & $0.63 \%$ & \\
\hline & & (3) & 0.1030 & 0.1050 & $-2.0 \mathrm{E}-03$ & & $-1.94 \%$ & \\
\hline & & (4) & 0.0868 & 0.0871 & $-3.0 \mathrm{E}-04$ & & $-0.35 \%$ & \\
\hline & & (5) & 0.1106 & 0.1110 & $-4.0 \mathrm{E}-04$ & & $-0.36 \%$ & \\
\hline & & (6) & 0.1057 & 0.1050 & $7.0 \mathrm{E}-04$ & & $0.66 \%$ & \\
\hline \multirow[t]{6}{*}{2} & & (1) & 0.1175 & 0.1180 & $-5.0 \mathrm{E}-04$ & $-3.67 \mathrm{E}-04$ & $-0.43 \%$ & $-0.31 \%$ \\
\hline & & (2) & 0.1046 & 0.1050 & $-4.0 \mathrm{E}-04$ & & $-0.38 \%$ & \\
\hline & & (3) & 0.0893 & 0.0889 & 4.0E-04 & & $0.45 \%$ & \\
\hline & & (4) & 0.1193 & 0.1190 & $3.0 \mathrm{E}-04$ & & $0.25 \%$ & \\
\hline & & (5) & 0.1020 & 0.1030 & $-1.0 \mathrm{E}-03$ & & $-0.98 \%$ & \\
\hline & & (6) & 0.1300 & 0.1310 & $-1.0 \mathrm{E}-03$ & & $-0.77 \%$ & \\
\hline \multirow[t]{6}{*}{3} & & (1) & 0.0895 & 0.0898 & $-3.0 \mathrm{E}-04$ & $-1.17 \mathrm{E}-04$ & $-0.34 \%$ & $-0.14 \%$ \\
\hline & & (2) & 0.0742 & 0.0743 & $-1.0 \mathrm{E}-04$ & & $-0.13 \%$ & \\
\hline & & (3) & 0.0925 & 0.0929 & $-4.0 \mathrm{E}-04$ & & $-0.43 \%$ & \\
\hline & & (4) & 0.0816 & 0.0813 & $3.0 \mathrm{E}-04$ & & $0.37 \%$ & \\
\hline & & (5) & 0.0703 & 0.0706 & $-3.0 \mathrm{E}-04$ & & $-0.43 \%$ & \\
\hline & $\therefore 0$ & (6) & 0.0853 & 0.0852 & $1.0 \mathrm{E}-04$ & & $0.12 \%$ & \\
\hline
\end{tabular}

Note: P: Point; RSMR: Root mean square roughness; AE: Absolute error; MAE: Mean absolute error; RE: relative error; MRE: Mean relative error.

Table 3. Calculation results of statistical index MTD of asphalt pavement texture parameters

\begin{tabular}{|c|c|c|c|c|c|c|c|}
\hline \multirow{3}{*}{$\mathrm{S}$} & \multicolumn{3}{|c|}{ MTD } & \multirow{2}{*}{\multicolumn{4}{|c|}{ Statistical Parameter }} \\
\hline & \multirow{2}{*}{$\begin{array}{c}\text { Benchmark I } \\
\text { Sand patch }\end{array}$} & \multirow{2}{*}{$\frac{\text { Benchmark II }}{\text { ZGScan }}$} & \multirow{2}{*}{$\begin{array}{c}\text { Validation } \\
\text { ACRP System }\end{array}$} & & & & \\
\hline & & & & $\mathrm{AE}(\mathrm{mm})(\mathrm{I})$ & $\mathrm{RE}(\mathrm{I})$ & $\mathrm{AE}(\mathrm{mm})(\mathrm{II})$ & $\mathrm{RE}(\mathrm{II})$ \\
\hline 1 & 0.94 & 0.9310 & 0.9265 & $1.6 \mathrm{E}-02$ & $1.72 \%$ & $4.5 \mathrm{E}-03$ & $0.48 \%$ \\
\hline 2 & 0.75 & 0.7675 & 0.7691 & $-1.7 \mathrm{E}-02$ & $-2.29 \%$ & $-1.6 \mathrm{E}-03$ & $-0.21 \%$ \\
\hline 3 & 0.88 & 0.8611 & 0.8670 & $1.5 \mathrm{E}-02$ & $1.67 \%$ & $-5.9 \mathrm{E}-03$ & $-0.69 \%$ \\
\hline 4 & 0.55 & 0.5350 & 0.5420 & 4.9E-03 & $0.90 \%$ & $-7.0 \mathrm{E}-03$ & $-1.31 \%$ \\
\hline 5 & 0.67 & 0.6710 & 0.6648 & $9.6 \mathrm{E}-03$ & $1.42 \%$ & $6.2 \mathrm{E}-03$ & $0.92 \%$ \\
\hline 6 & 0.64 & 0.6129 & 0.6051 & $3.1 \mathrm{E}-02$ & $4.83 \%$ & $7.8 \mathrm{E}-03$ & $1.27 \%$ \\
\hline 7 & 0.49 & 0.4790 & 0.4811 & $8.4 \mathrm{E}-03$ & $1.72 \%$ & $-2.1 \mathrm{E}-03$ & $-0.44 \%$ \\
\hline 8 & 0.45 & 0.4403 & 0.4446 & 8.7E-03 & $1.91 \%$ & $-4.3 \mathrm{E}-03$ & $-0.98 \%$ \\
\hline
\end{tabular}

Note: S: Road section.

The data in Table 3 shows that the ACPR system has high measurement accuracy and the relative error (RE) is within $\pm 5 \%$, based on the MTD values obtained by the sand patch method and ZGScan, respectively. In addition, the MTD values obtained by the ZGScan and ACRP system are smaller than that of the sand patch 
method. This is because the laser measurement and the close range photogrammetry are all based on the principle of light propagation which cannot capture the partially hidden curved tunnel and fine seams of the asphalt mixture during the data acquisition process. Therefore, the calculated filling volume of the imaginary sand is smaller. The sand patch method is to fill the pavement texture with a fixed volume of test sand, which can better measure the MTD value of the close-graded pavement.

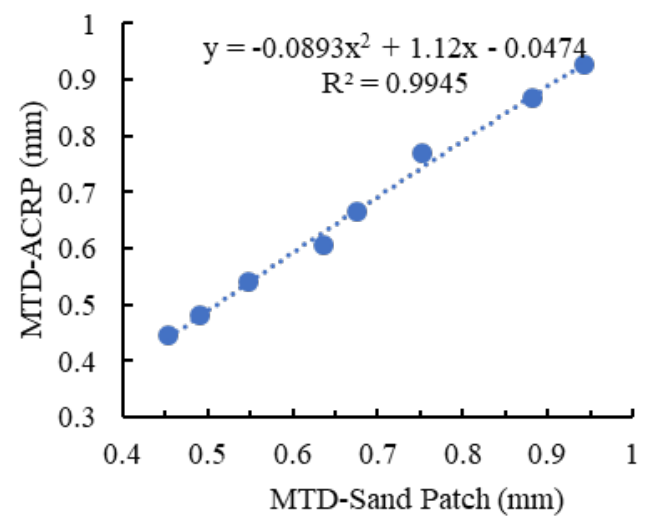

Fig. 7. Correlation analysis between the results of the sand patch method and the ACRP system measurement
The correlation analysis between the results of the sand patch method and the ACRP system measurement is shown in Fig. 7. After eliminating the abnormal data, $R^{2}$ reaches 0.9945 , which indicates that the ACRP system proposed in this paper can collect high-precision asphalt pavement texture, and can replace the sand patch method to collect the pavement texture MTD value.

\subsection{High efficiency of close range photogrammetry system}

Statistical analysis was carried out on the time taken to collect texture information by ACRP system, ZGScan and sand patch method. The interval of the reconstructed cloud points was set to be $0.01 \mathrm{~mm}$, and the test time of single-point sand patch was recorded. The statistical data is shown in Fig. 8. The data shows that the time ACRP system consumed to obtain the surface texture of the asphalt pavement and calculate the texture parameters is much less than that of the ZGScan and the traditional sand patch method, which means ACRP system can efficiently complete the surface texture parameter measurement of the asphalt pavement.

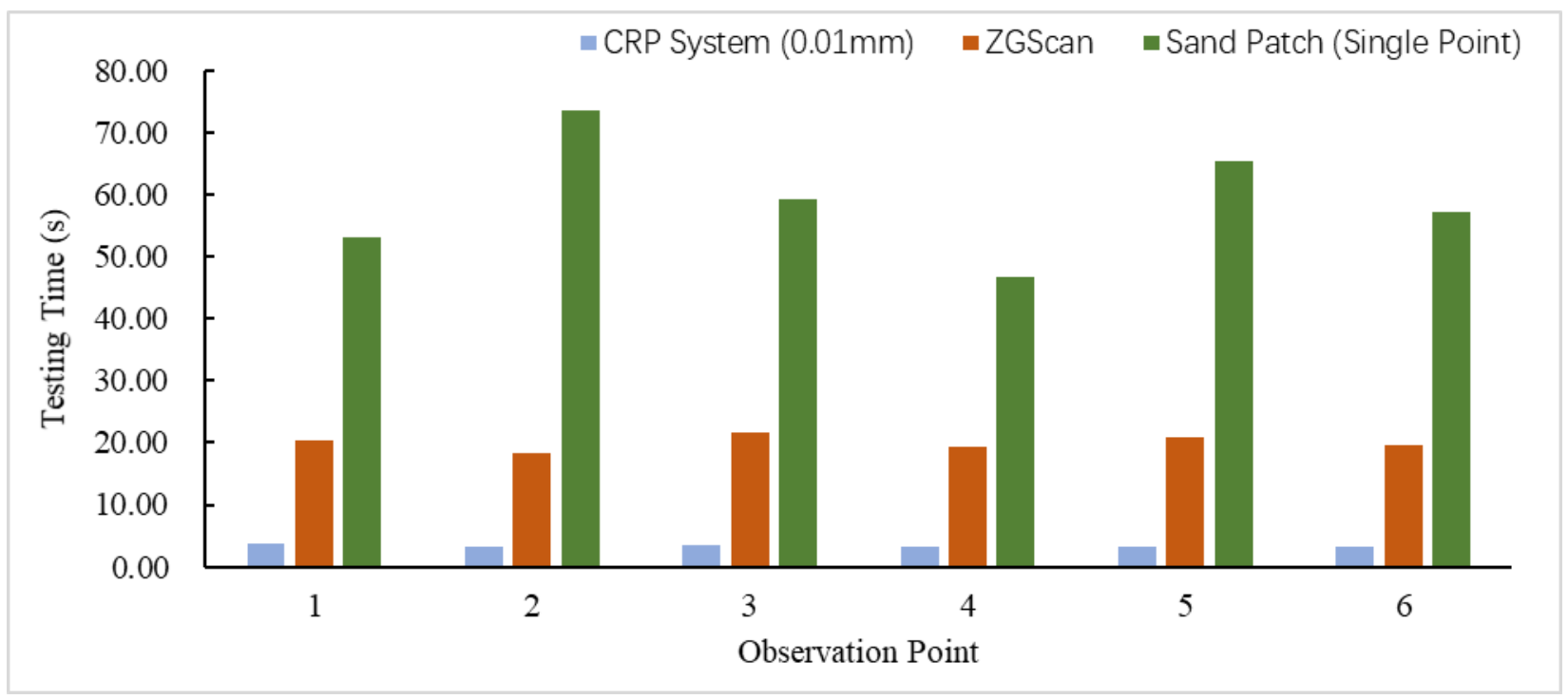

Fig. 8. Time consumption of each test method

\section{Conclusion}

(1) The ACRP platform determines the orientation and relative position of the three cameras, and can solve the coordinates of spatial points by using the camera external parameters $R, T$ matrix as known parameters. Compared with the traditional close range photogrammetry technology, there is no need to solve the external parameters $R 、 T$ matrix, which improves the efficiency of acquisition and measurement. 
(2) The ACPR system is used to test the asphalt pavement texture information on site, and the obtained result is compared with those of laser measurement (ZGScan) and sand patch method. The MRE values are all within $\pm 0.5 \%$, and the RE values are all within $\pm 5 \%$. The RSMR and MTD indicators obtained by the ACRP system can achieve the accuracy requirement of the ZGScan handheld industrial laser scanner.

(3) Through the correlation analysis, the MTD value extracted by the ACRP system has a good linear correlation with that of the sand patch method. Compared with the sand patch method, the MTD obtained by the ZGScan and CRP systems are smaller.

In summary, the ACRP system based on CRP technology proposed in this paper can collect high-precision asphalt pavement texture efficiently in real time, which greatly improves the working efficiency and accuracy of traditional close range photogrammetry. It can provide subsequent driverless vehicle braking studies with real-time and efficient pavement texture parameters.

\section{Acknowledgements}

The study is financially supported by National Natural Science Foundation of China (No.51778139) and the Research and Innovation Foundation for Graduate Students in Jiangsu Province of China (No. KYCX18_0146).

\section{References}

1. UECKERMANN A, WANG D, OESER M, et al. Calculation of skid resistance from texture measurements[J]. Journal of Traffic and Transportation Engineering(English Edition). 2015, 2: 3-16.

2. KOGBARA R B, MASAD E A, WOODWARD D, et al. Relating surface texture parameters from close range photogrammetry to Grip-Tester pavement friction measurements $[\mathrm{J}]$. Construction \& Building Materials. 2018, 166: 227-240.

3. SUN L, WANG Y Three-Dimensional Reconstruction of Macrotexture and Microtexture Morphology of Pavement Surface Using Six Light Sources-Based Photometric Stereo with Low-Rank Approximation[J].
Journal of Computing in Civil Engineering. 2016, 31: 04016054.

4. Wang Duanyi, LiWeijie, Zhang Xiaoning. Evaluation and Measurement of Surface Depth of Asphalt Road Using Digital Image Technology[J]. Journal of South China University of Technology(Natural Science Edition). 2004, 32: 42-45. (in Chinese)

5. Zhang Xiaoning, Duan Yuehua, Lizhi, et al. Classification of Asphalt Mixture Materials Based on X-ray CT[J]. Journal of South China University of Technology(Natural Science Edition). 2011, 39: 120-124. (in Chinese)

6. Wang Duanyi, Qian Chaoqing. Research on Texture Characteristics of Asphalt Road Surface Based on Industrial CT Technology[J]. Science Technology and Engineering. 2016, 16: 270-274. (in Chinese)

7. VILAçA J L, FONSECA J C, PINHO A C M, et al. 3D surface profile equipment for the characterization of the pavement texture - TexScan[J]. Mechatronics. 2010, 20: 674-685.

8. MCGOWAN R, FEIGHAN K, MULRY B, et al. Use of Close range Photogrammetry to Characterize Texture in a Pavement Surfacing Material[J]. 2018.

9. SUŽIEDELYTĖ-VISOCKIENĖ J Accuracy analysis of measuring close range image points using manual and stereo modes[J]. Geodesy and Cartography. 2013, 39: 18-22.

10. GRANSHAW S I Close Range Photogrammetry: Principles, Methods And Applications[J]. Photogrammetric Record. 2010, 25: 203-204.

11. LUHMANN T, ROBSON S, KYLE S, et al. 2013. Close range photogrammetry and $3 \mathrm{D}$ imaging $[\mathrm{M}]$. Walter de Gruyter.

12. HOU Z, WANG K C P, GONG W 2007. Experimentation of 3D Pavement Imaging through Stereovision [C] //; City. 376-381.

13. MACHIKHIN A S, GOREVOY A V 2016. Calibration of miniature prism-based stereoscopic imagers for precise spatial measurements [C] //, International Society for Optics and Photonics; City. 991707.

14. GORDON I, LOWE D G What and Where: 3D Object Recognition with Accurate Pose[J]. 2006, 4170: 67-82. 